\title{
Change in attentional control predicts change in attentional bias to negative information in response to elevated state anxiety
}

\author{
Julian Basanovic, Isabelle Kaiko, Colin MacLeod \\ Centre for the Advancement of Research on Emotion, \\ School of Psychological Science \\ The University of Western Australia
}

Corresponding author:

Julian Basanovic

julian.basanovic@uwa.edu.au

\section{Acknowledgements:}

The research reported in this manuscript was supported by the Australian Research Council (FL170100167).

\section{Conflict of Interest:}

The authors declare that they have no conflict of interest

Keywords:

anxiety; state anxiety; attentional bias; attentional control

\section{Publication:}

This is a post-peer-review, pre-copyedit version of an article published in Cognitive Therapy and Research. The final authenticated version is available online at:

https://doi.org/10.1007/s10608-020-10176-3

Basanovic, J., Kaiko, I., \& MacLeod, C. (2020). Change in Attentional Control Predicts Change in Attentional Bias to Negative Information in Response to Elevated State Anxiety. Cognitive Therapy and Research. https://doi.org/10.1007/s10608-020-10176-3 


\title{
Change in attentional control predicts change in attentional bias to negative information in response to elevated state anxiety
}

\begin{abstract}
Background: Researchers have demonstrated that elevation in state anxiety leads to elevation in attentional bias favouring the processing of negative information, and that the magnitude of this attentional bias change varies across individuals. However, research has not identified the mechanisms that underpin individual variation in state-anxiety induced attentional bias change. Researchers have also demonstrated that inhibitory control of attention becomes impaired when state anxiety is elevated, and cognitive models propose that impaired inhibitory control of attention may underpin attentional bias to negative information. Thus, the present study investigated whether individual differences in the magnitude of attentional bias elevation elicited by heightened state anxiety is predicted by the degree to which such state anxiety elevation impairs attentional control.
\end{abstract}

Methods: Eighty participants completed assessments of attentional bias to negative information and inhibitory control of attention prior to, and following, a procedure designed to elevate state anxiety.

Results: It was observed that greater elevation in attentional bias to negative information was predicted by lesser decline in inhibitory control of attention as state anxiety increased.

Conclusions: Findings support proposal of a relationship between attentional control and attentional bias to negative information, though are inconsistent with the proposal that heightened attentional bias to negative information is uniformly underpinned by greater impairment in attentional control. Implications are discussed. 


\section{Introduction}

Heightened attentional bias to negative information, reflecting a relatively heightened attentional preference for the processing of negative information over nonnegative information, is implicated as a cognitive characteristic of heightened anxiety vulnerability (Bar-Haim, Lamy, Pergamin, Bakermans-Kranenburg, \& van IJzendoorn, 2007). Attentional bias to negative information is commonly assessed using the dot-probe task. In this task, participants are briefly presented with pairs of emotionally discrepant stimuli. One stimulus is negatively valenced in emotional tone (e.g., the word "kill") while the other is non-negatively valenced (e.g., the word "hair"). Following offset of the stimulus pair, participants must discriminate the identity of a probe that appears in a location previously occupied by one of the two stimuli. It is assumed that individuals will be quicker to discriminate probes appearing in attended locations, and so an index of attentional bias to negative information is derived by computing relative speeding to discriminate probes presented in the location of negative stimuli as compared non-negative stimuli. Using this index of attentional bias researchers have reported that elevated state anxiety is associated with heightened attentional bias to negative information (Bar-Haim et al., 2007).

Studies demonstrating that elevated state anxiety is accompanied by heightened attentional bias to negative information have sometimes measured naturally occurring variation in state anxiety (MacLeod \& Mathews, 1988; Mogg, Bradley, De Bono, \& Painter, 1997), but also have experimentally manipulated state anxiety (Liu, Shen, \& Li, 2019; Mogg, Mathews, Bird, \& Macgregor-Morris, 1990). For example, Mogg et al. (1990) subjected high and low trait anxious participants to a lab-based induction designed to elevate state anxiety (receiving a negative evaluation of performance), or a condition that did not induce elevation in state anxiety (receiving a positive evaluation of performance). The investigators 
observed that the induction procedure elevated state anxiety as intended, and that the induction also resulted in heighted attentional bias to negative words as compared to the condition that did not induce elevation in state anxiety. Though high trait anxious individuals do not necessarily exhibit an attentional preference for negative information over nonnegative information (Kruijt, Parsons, \& Fox, 2019), the degree to which elevated state anxiety leads to heightening of attentional bias to negative information has been shown to be enhanced amongst individuals with heightened anxiety vulnerability. For example, when MacLeod and Mathews (1988) examined the impact of elevated state anxiety on attentional bias to negative information in high and low trait anxious participants it was observed that high trait anxious participants demonstrated a disproportionate state anxiety elicited increase in attentional bias to negative information.

Though research has demonstrated that elevated state anxiety is characterised by elevation in attentional bias to negative information the cognitive mechanisms underpinning this effect have not been empirically demonstrated. Therefore, it is not known why individuals differ in the degree to which elevated state anxiety elicits elevation in attentional bias to negative information. Identification of these mechanisms will not only advance understanding of the association between state anxiety and attentional bias but may also reveal appropriate targets for cognitive interventions designed to attenuate heightened attentional bias to negative information.

Prominent theories of selective attention identify impaired cognitive control as a mechanism that enhances interference from distractor stimuli during selective attention (Lavie, Hirst, de Fockert, \& Viding, 2004; Murphy, Groeger, \& Greene, 2016). Given the proposed interplay between cognitive control processes and selective attention, theorists have proposed that poor inhibitory control of attention may contribute to attentional bias to 
negative information. Inhibitory control of attention, reflecting the capacity to which individuals can inhibit attentional capture by stimuli in favour of allocating attention in accordance with attentional goals, falls within the collection of inhibitory control processes proposed to underpin executive function (Miyake et al., 2000). Attentional Control Theory proposes that inhibitory control of attention can serve to diminish prepotent attentional capture of emotional distracters (Eysenck, Derakshan, Santos, \& Calvo, 2007). Similarly, Cisler and Koster (2011) proposed impaired attentional control to be a mechanism facilitating anxiety-linked attentional bias to negative information. These proposals have received some empirical support. For example, researchers have demonstrated that the application of transcranial electro-stimulation to enhance attentional control can result in attenuation of attentional bias to negative information (Chen, Basanovic, Notebaert, MacLeod, \& Clarke, 2017). When considering research involving anxiety vulnerability, it has also been shown that individuals with higher levels of trait anxiety are characterised by poorer inhibitory control of attention, when compared to individuals lower in trait anxiety (Ansari \& Derakshan, 2010; Basanovic et al., 2018), and that elevated state anxiety can serve to impair inhibitory control of attention (Bishop, Duncan, Brett, \& Lawrence, 2004; PachecoUnguetti, Acosta, Callejas, \& Lupiáñez, 2010).

Importantly, though research has examined the general association between measures of attentional control and attentional bias to negative information, research has yet to empirically investigate their association in the context of changes in state anxiety. It is plausible that variation in the degree to which elevated state anxiety increases attentional bias to negative information may reflect individual differences in the degree to which elevated state anxiety compromises inhibitory control of attention. If this is the case, then measures of the degree to which attentional bias to negative information increases in 
response to elevated state anxiety should be predicted by measures of the degree to which inhibitory control of attention was compromised in response to the same state anxiety elevation.

Thus, the aim of the present study was to determine the validity of the hypothesis that variation in the degree to which heightened state anxiety leads to elevation in attentional bias to negative information is predicted by variation in the degree to which heightened state anxiety leads to impairment of inhibitory control of attention. Participants completed tasks that assessed attentional bias to negative information and a task that assessed inhibitory control of attention, both prior to and following a procedure designed to induce elevation in state anxiety. Analyses examined whether variation in the degree to which elevation in state anxiety led to heightened attentional bias to negative information was predicted by variation in the degree to which elevation in state anxiety led to the impairment of inhibitory control of attention.

\section{Method}

\section{Participants}

Participants were recruited from a cohort of 917 undergraduate students at the University of Western Australia who had completed the trait scale of the Spielberger StateTrait Anxiety Inventory (STAI-T) earlier in the university semester. A power analysis determined that 80 participants would provide $80 \%$ power $(1-\beta=0.80)$ to detect a correlation of $r>.30$ between two measures, with a two-tailed test of statistical significance set at $p \leq .05$. To allow examination of the potential influence of trait anxiety, 40 participants who had scored in bottom-third (25 Female; Age, $M=20.50, S D=5.73 ;$ STAI-T, $M=33.30$, $S D=5.58$ ) and 40 who had scored in the top third (25 Female, 2 non-binary; Age, $M=20.80$, 
$\mathrm{SD}=4.90 ; \mathrm{STAI}-\mathrm{T}, \mathrm{M}=54.5, \mathrm{SD}=6.49)$ of the distribution of scores from the undergraduate cohort were recruited.

\section{Materials}

\section{Apparatus}

All assessments were delivered using a computer and 22-inch monitor with a $15 \mathrm{~ms}$ refresh rate. Participant responses were made using a standard USB mouse and keyboard.

\section{Trait Anxiety Inventory}

The trait anxiety assessment scale of the Spielberger State-Trait Anxiety Inventory (STAI-T; Spielberger, Gorsuch, Lushene, Vagg, \& Jacobs, 1983) was used to assess participants' levels of trait anxiety. Scores on the STAI-T can range from 20 to 80, with higher scores representing higher levels of trait anxiety.

\section{State Anxiety Inventory - Short Form}

The six-item short form of the state anxiety assessment scale of the Spielberger State-Trait Anxiety Inventory (Marteau \& Bekker, 1992) was used to assess participants' levels of state anxiety throughout the experiment session. In order to allow for a more precise reporting of change in state anxiety than can be achieved using a four-point ordinal scale, participants responded to each item using a visual analogue scale that ranged from 0 40, with higher points on the scale indicating stronger state anxiety symptomatology. Thus, scores on the SAI- 6 could range from 0 to 240, with higher scores representing higher levels of state anxiety.

\section{Attentional Bias Assessment Task}

The attentional bias assessment task employed a dot-probe methodology that assessed attentional bias to negative information by measuring the time taken for 
participants to discriminate visual probes presented either in the location of negative information or non-negative information. The task comprised two blocks of 48 trials each.

All trials were presented on a black background. Each trial started with the presentation of a fixation cue, $8 \mathrm{~mm}$ in height, in the centre of the screen for $1000 \mathrm{~ms}$. This cue was then removed, and a word-pair was presented. Each word was presented in white monospaced text $8 \mathrm{~mm}$ in height. Each word was presented $12 \mathrm{~mm}$ above, or below, the location of the initial fixation cue. In each pair, one word was negatively valenced in emotional tone (e.g. "FAILURE"), and the other was an equivalent length word that was nonnegative in tone (e.g. "BOTTLES") ${ }^{1}$. The negative word randomly appeared in each location with equal frequency across trials. After $500 \mathrm{~ms}$, the word-pair disappeared, and a small target probe was presented in the location previously occupied by one of the words. This probe appeared equally often in the location previously occupied by the negative word and the non-negative word. The probe was a grey arrow, $4 \mathrm{~mm}$ long, pointing either upward or downward with equal frequency across trials. Participants were required to discriminate the orientation of the probe by rapidly pressing the corresponding arrow key on the keyboard. Incorrect responses triggered a 3-second error message. At the conclusion of each trial the latency and accuracy of participant's response was recorded and the next trial commenced after a $1000 \mathrm{~ms}$ inter-trial-interval.

At the conclusion of the task, an index was derived from the difference, in milliseconds, between the mean latency at which the participant discriminated probes appearing in the location of negative words as compared to non-negative words. Greater relative speeding to discriminate probes appearing in the location of negative words was

\footnotetext{
${ }^{1}$ Word pairs used in this task can be found in the supplementary material associated with this manuscript.
} 
believed to reflect greater attention allocation to negative stimuli across trials. Thus, a greater positive score on this Attentional Bias Index indicated greater attentional bias to negative information.

\section{Inhibitory Control of Attention Assessment Task}

The inhibitory control of attention assessment task adopted an attentional-probe methodology previously demonstrated capable to detecting associations between individual differences in attentional control and biases in attentional processing (Basanovic et al., 2017), and that mimics tasks commonly employed by researchers to assess this process (e.g. Ansari \& Derakshan, 2010, 2011; Cornwell, Mueller, Kaplan, Grillon, \& Ernst, 2012;

Derakshan et al., 2009; Friedman \& Miyake, 2004; Miyake et al., 2000). This task was designed to measure time taken to execute attentional shifts that either require, or do not require, inhibitory control of attention. The task comprised three types of trials, noninhibitory attentional shift trials, inhibitory attentional shift trials, or no attentional shift (baseline) trials. The task comprised two blocks of 72 trials each, with each trial type presented with equal frequency.

All trials were presented on a black background. Trials started with the presentation of a single word cue, $8 \mathrm{~mm}$ in height, in the centre of the screen for $1000 \mathrm{~ms}$. This cue informed participants of the attentional response that would be required on the trial. A blank screen was presented for 2000 ms following this cue. What happened afterward depended on trial type as described next.

On non-inhibitory, and inhibitory, attentional shift trials a visually salient stimulus (bright white circle outline, $80 \mathrm{~mm}$ in diameter and $5 \mathrm{~mm}$ thick) appeared abruptly on the left or right side of the cue. On non-inhibitory attentional shift trials the cue was "ATTEND", indicating that the participant should attend to the subsequently presented lateral circle. 
The visual probe was simultaneously presented within the circle, and so the attentional shift needed to identify probes on this trial did not require inhibiting attention to this salient bright shape. On inhibitory attentional shift trials the cue was "AVOID", indicating that the participant should inhibit attention to the subsequently presented lateral circle and move attention to the opposite side of the screen. The probe appeared in the opposite side of the screen to the circle, and so the attentional shift needed to identify this probe required inhibiting attention to this salient bright shape. On baseline trials the cue was "CENTRE", indicating that attention should remain upon the cue location. The visual probe appeared, either alone or within a centrally positioned circle, in the same location as the cue and so no attentional shift was required to be executed.

On all trials the target probe was a grey arrow, $4 \mathrm{~mm}$ long, pointing upward or downward with equal frequency across trials. Participants were required to discriminate the orientation of the probe by rapidly pressing the corresponding arrow key on the keyboard. Incorrect responses triggered a 3-second error message. At the conclusion of each trial the latency and accuracy of participant's response was recorded and the next trial commenced after a $1000 \mathrm{~ms}$ inter-trial-interval.

At the conclusion of the task an index was derived from the difference, in milliseconds, between the latency at which participants shifted attention away from the salient bright circle as compared to towards the circle. First, the latency to execute each attentional shift, independent of the time taken to discriminate probe orientation, was revealed by computing the slowing, in milliseconds, to discriminate probes during noninhibitory, or inhibitory, attentional shift trials as compared to probes during baseline trials. Next, the relative difference in each attentional shift latency was computed. These steps are expressed via the following equation: 
Inhibitory Attentional Control Cost Index =

[Mean Latency:Inhibitory attentional shift trials - Mean Latency:Baseline trials without circle]

- [Mean Latency:Non-inhibitory attentional shift trials -Mean Latency:Baseline trials with circle]

Greater relative slowing to shift attention during Inhibitory attentional shift trials, as compared to Non-inhibitory attentional shift trials, was believed to reflect greater attentional "cost" when inhibitory control of attention was required. Thus, a greater positive score on this Inhibitory Attentional Control Cost Index reflected poorer inhibitory control of attention.

\section{State Anxiety Induction}

A state anxiety induction procedure was implemented, during the latter half of the experimental session, to elicit elevated state anxiety. This induction procedure involved informing participants that they would be required to complete an 'interview performance evaluation task', in which they must engage in a simulated job interview by responding to questions asked by a pre-recorded 'hiring panel' while their own interview performance was recorded on video for later evaluation by other students. This state anxiety induction procedure has demonstrated success at producing elevation in state anxiety amongst participants in previous studies conducted within our research group, and recent scrutiny of affect induction procedures has demonstrated efficacy of this kind of approach in elevating state anxiety (Joseph et al., 2020).

\section{Procedure}

On arrival participants provided informed consent. Participants next completed practice trials before completing two blocks of the Attentional Bias Assessment Task and 
Attentional Control Assessment Task in an alternating order counterbalanced across participants. The STAIS- 6 was administered prior to and following each trial block. Next, participants were exposed to the state anxiety induction by being provided with a second information sheet informing them for the first time that the interview evaluation task would immediately follow a second completion of these attentional tasks. Participants next completed the Attentional Bias Assessment Task and Attentional Control Assessment Task in the same order as before, this time believing. Again, the STAIS- 6 was administered prior to and following each trial block. Upon completion of the tasks participants were debriefed.

\section{Results}

Analyses first determined whether the state anxiety induction procedure succeeded in elevating state anxiety. Next, the impact of state anxiety conditions upon attentional bias to negative information and inhibitory control of attention was examined. Finally, the main aim of the study was addressed by determining whether variation in the magnitude of change in attentional bias to negative information elicited by the elevation of state anxiety was predicted by variation in the magnitude of change in inhibitory control of attention elicited by the elevation of state anxiety. Descriptive statistics for measures of state anxiety and attentional processes are presented in Table 1.

\section{Data preparation}

Attentional indices for each attention assessment task were computed after excluding latencies that resulted from incorrect probe discrimination responses, that were below 200 ms or above 2000 ms, and remaining latencies that fell more than 2.58 SD from the participant's mean latency under each critical trial condition, in line with prior research (Rudaizky, Basanovic, \& MacLeod, 2014). This resulted in 4.51\% of attentional biases 
assessment trial latencies, and 5.22\% of attentional control assessment trial latencies, excluded from the computation of index scores.

Upon reviewing the attentional bias index scores computed across participants it was discovered that three participants exhibited index scores greater than 3 standard deviations from the mean of all other attentional bias index scores. As such, these three participants were excluded from descriptive statistics and analyses that involved attentional bias index scores.

\section{Effectiveness of State Anxiety Induction}

Participants' level of state anxiety experienced before and after exposure to the state anxiety induction procedure, was expressed as the mean of participants' SAI- 6 questionnaire scores recorded before and after the state anxiety induction procedure. To determine whether the state anxiety induction procedure succeeded in elevating state anxiety, these scores were subjected to a mixed-design ANOVA analysis, that considered Assessment Point (Pre State Anxiety Induction, Post State Anxiety Induction) as a withingroups factor, and Trait Anxiety Group (Low Trait Anxiety Group, High Trait Anxiety Group) as a between-groups factor. The analysis revealed the anticipated main effect of Assessment Point, $F(1,78)=41.77, p<.001, \eta^{2} p=0.35$, reflecting the fact that, on average, participants reported higher levels of state anxiety following exposure to the intended state anxiety induction $(M=90.37, S D=42.99)$, than they reported prior to this state anxiety induction ( $M$ $=71.92, \mathrm{SD}=38.50)$. Unsurprisingly, the analyses also revealed a main effect of Trait Anxiety Group, $F(1,78)=26.1, p<.001, \eta^{2} p=0.25$, reflecting the fact that, on average, participants in the High Trait Anxiety Group reported higher levels of state anxiety ( $M=100.43, S D=37.32$ ) than did those in the Low Trait Anxiety Group ( $M=61.85,36.83)$. The interaction effect between these factors did not approach significance $(p=0.33)$, meaning that the state 
anxiety induction was equally effective in successfully elevating state anxiety for both groups of participants. The pattern of data that gave rise to these effects is illustrated in Figure 1.

\section{Effect of State Anxiety upon Attentional Bias and Attentional Control}

To determine whether this induced state anxiety elevation served to impair inhibitory control of attention, a mixed design ANOVA was conducted on Inhibitory Attentional Control Cost Index scores. This analysis again considered Assessment Point (Pre State Anxiety Induction, Post State Anxiety Induction) as a within-groups factor, and Trait Anxiety Group (Low Trait Anxiety Group, High Trait Anxiety Group) as a between-groups factor. The main effect of Assessment Point was statistically significant, $F(1,78)=3.95, p$ $=.050, \eta^{2} p=0.05$, reflecting that, on average, participants demonstrated larger Inhibitory Attentional Control Cost Index scores following the state anxiety induction ( $M=110.31, S D=$ $84.88)$ than they had shown prior to the state anxiety induction $(M=91.40, S D=78.85)$. No other significant effects emerged from the analysis (smallest $p=0.32$ ). Thus, the obtained results confirm that, for both high and low trait anxious participants, inhibitory control of attention was reduced when state anxiety was elevated by the induction procedure. The pattern of data that gave rise to these effects is illustrated in Figure 2.

To determine whether the induced state anxiety elevation led to heighted attentional bias to negative information, an ANOVA of the same design was conducted on the Attentional Bias Index scores. The main effect of Trait Anxiety Group was statistically significant, $F(1,75)=4.58, p=.036, \eta^{2} p=0.06$, reflecting that, on average, participants in the Hight Trait Anxiety Group demonstrated larger Attentional Bias Index scores score $(M=5.03$, $S D=20.16)$ as compared to participants in the Low Trait Anxiety Group ( $M=-1.56, S D=$ 18.13). No other significant effects emerged from the analysis (smallest $p=0.21$ ). Thus, though this analysis revealed evidence of trait anxiety-linked attentional bias to negative 
information, it did not reveal evidence that the experimentally induced elevation of state anxiety served to modify attentional bias to negative information. The pattern of data that gave rise to these effects is illustrated in Figure 3.

Following these analyses, the internal reliability of the attention assessment tasks was computed. These were computed using a Spearman-Brown corrected split-half permutation

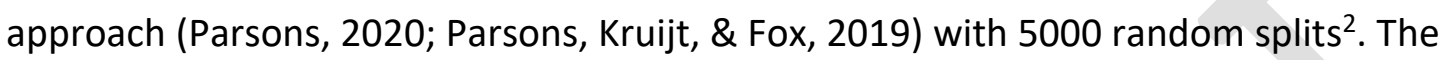
estimated internal consistency of Attentional Bias Index scores prior to the state anxiety induction was $r_{\mathrm{SB}}=-.26, \mathrm{Cl}_{95 \%}[-.57, .10]$, and following the state anxiety induction was $r_{\mathrm{SB}}$ $=.21, \mathrm{Cl}_{95 \%}[-.12, .48]$. The estimated internal consistency of Attentional Control Index scores prior to the state anxiety induction was $r_{\mathrm{SB}}=.59, \mathrm{Cl}_{95 \%}[.41, .73]$, and following the state anxiety induction was $r_{\mathrm{SB}}=.61, \mathrm{Cl}_{95 \%}[.29, .77]$.

\section{Examination of the Association between State Anxiety Elicited Change in Attentional Bias and Change in Attentional Control}

The fact that inducing elevated state anxiety did not elicit a general increase in attentional bias to negative information does not preclude the possibility that variation across participants in the degree to which it did so could be a function of the degree to which state anxiety elevation served to impair attentional control. To determine whether this was the case, two scores were computed for each participant. One score, labelled Inhibitory Attentional Control Cost Increase, reflected the amount the participant's Inhibitory Attentional Cost Index score increased following the state anxiety induction, with higher scores meaning that the elevation of state anxiety had led to greater reduction in

\footnotetext{
${ }^{2}$ For readers interested in a traditional split-half approach, the correlation between $1^{\text {st }}$ block and $2^{\text {nd }}$ block trials for each assessment task was as follows. $\mathrm{AB}$ (Pre-Induction) $r_{\mathrm{SB}}=-.08, \mathrm{AB}$ (Post-Induction) $r_{\mathrm{SB}}=.37, \mathrm{AC}(\operatorname{Pre}$ Induction) $r_{\mathrm{SB}}=.51, \mathrm{AC}$ (Post Induction) $r_{\mathrm{SB}}=.41$.
} 
inhibitory control of attention. The other score, labelled Attentional Bias Increase, reflected the amount the participant's Attentional Bias Index score increased following the state anxiety induction, with higher scores meaning that the elevation of state anxiety had led to greater increase in attentional bias to negative information.

To examine whether variation in state anxiety-linked change in attentional bias to negative information was predicted by change in attentional control, a linear regression was conducted. This regression included Attentional Bias Increase scores as the outcome variable and Inhibitory Attentional Control Cost Increase scores as the predictor variable. The model determined that, as anticipated, Attentional Bias Increase scores were significantly predicted by Inhibitory Attentional Control Cost Increase scores, $F(1,75)=6.83$, $b=-0.10, \mathrm{SE}=0.04,6=-0.29, p=.011, R^{2}=.08$. Thus, the model determined that greater state anxiety elicited elevation of attentional bias to negative information was predicted by lesser state anxiety elicited diminishment in inhibitory control of attention. An illustration of the relationship between these variables is present in Figure 4. While this supported the prospect that variation in state anxiety elicited change in attentional control may contribute to variance in state anxiety elicited change in attentional bias, the direction of this association was inverse to the direction proposed by the hypothesis under test.

\section{Discussion}

The aim of the present study was to determine whether variation in the degree to which heightened state anxiety leads to elevation in attentional bias to negative information is predicted by variation in the degree to which heightened state anxiety leads to impairment of inhibitory control of attention. The procedure adopted to induce elevated state anxiety was found to do so effectively, and this elevation of state anxiety served to generally compromise inhibitory control of attention, though did not lead to a general 
increase in attentional bias to negative information, across participants. Importantly, crossparticipant variation in the degree to which elevated state anxiety resulted in elevation in attentional bias to negative information was predicted by cross-participant variation in the degree to which elevated state anxiety served to impair inhibitory control of attention. Contrary to expectation, across participants greater levels of state anxiety elicited elevation of attentional bias to negative information was predicted by lesser levels of state anxiety elicited decrement in inhibitory control of attention.

Within present study, the absence of evidence to demonstrate a general impact of elevated state anxiety upon attentional bias to negative information is inconsistent with previous research that has observed elevated state anxiety to lead to a general increase in attentional bias to negative information (Liu et al., 2019; Mogg et al., 1997, 1990). In contrast, the present demonstration that elevated state anxiety lead to a general diminishment in attentional control is consistent with previous studies that have observed a general impact of elevated state anxiety upon attentional control performance (PachecoUnguetti et al., 2010).While the relation of the present findings with previous literature is noteworthy, it is also important to note that the presence or absence of these general effects do not impact the determination of whether variation in state anxiety linked change in each attentional process is associated.

Importantly, the present findings support the proposal made by theorists that variation in attentional control may contribute to variation in attentional bias to negative information (Cisler \& Koster, 2011; Eysenck et al., 2007). However, at least with respect to state anxiety induced change in these processes, the present findings demonstrate an association that contrasts with the proposal that attentional bias to negative information is underpinned by poor attentional control. Given inhibitory control of attention reflects the 
capacity to direct attention in line with task goals the present finding leads to speculation that increased attentional bias to negative information in response to elevated state anxiety may represent an attentional goal rather than an attentional failure, and that greater inhibition of goal irrelevant (i.e. non-negative) information will allow greater success sustaining this attentional goal. Though some models of attentional bias to negative information have posited that contexts that elevate anxious mood states may encourage controlled selective attentional vigilance for negative stimuli (Cisler \& Koster, 2011; Mogg \& Bradley, 1998), the exact influence of state anxiety upon attentional control and attentional bias to negative information is not clearly illuminated by these models and future research will be necessary to clarify this tripartite relationship.

The present findings would be well considered in the context of findings that indicate influence of context on the flexible adjustment of attentional preference for stimuli. For example, an individual anxious about walking along a dark street at night may find their attention heightened, and more readily captured by signs of danger. Examining the influence of contextual change upon attention to emotional stimuli, Godara et al. (2020) observed that dysphoric individuals more readily switched attention goals to facilitate attention to negative information when that aligned with contextual goals. Other researchers have also observed influence of information processing goals on flexible attentional capture by stimuli relevant to these goals (Vogt, De Houwer, \& Moors, 2011; Vogt, De Houwer, Moors, Van Damme, \& Crombez, 2010). Research could usefully seek to determine the contribution of attentional flexibility and attentional goal setting to attentional processing of negative information in contexts that manipulate state anxiety.

Though the present study observed an effect of trait anxiety upon attentional bias to negative information, it did not observe a change in attentional bias to negative information 
across participants between state anxiety assessment points. The absence of an observed impact of state anxiety elevation upon measures of attentional bias to negative information may be the result of poor psychometric reliability of mean-based measure yielded by the dot-probe task employed in this study (MacLeod, Grafton, \& Notebaert, 2019). Researchers have noted the detrimental epistemic consequences arising from poor reliability of central tendency-based measures of attentional bias that are typically derived from the dot-probe task, particularly when examining within-individual variation (MacLeod et al., 2019; Van Bockstaele et al., 2019). In response, some theorists have advocated that measuring withinindividual variability in biased attention across an assessment task may more aptly measure anxiety-linked attentional bias to negative information (Zvielli, Bernstein, \& Koster, 2015), and recent evidence has implicated attentional control in the relationship between attentional bias variability and anxiety (Clarke et al., 2020). Future research examining the role of attentional control in state anxiety-linked change in attentional bias would benefit from measures shown to assess attentional bias to negative information precisely and reliably.

It is also important to consider that the present study subjected individuals to an induction designed to acutely elevate state anxiety. However, other researchers have examined change in attentional bias under conditions of more persistent elevation in state anxiety, such temporally distal, or proximal, to a major examination (MacLeod \& Rutherford, 1992). Longer-term environmental stressors (e.g. impending exams) may impact biases in selective attention, or attentional control, differently amongst individuals as compared circumstances of acute elevation in state anxiety (Mogg \& Bradley, 1998). Thus, future research could usefully examine whether the association between variation in change in 
attentional bias to negative information and attentional control remains when elevated state anxiety is induced via prolonged environmental stressors.

Further, it is noteworthy that though the present study demonstrates an association between variation in change in inhibitory control of attention and change in attentional bias to negative information in response to elevated state anxiety, it does not establish a causal association. Thus, future research could usefully determine whether manipulation of inhibitory control of attention change experienced in response to elevated state anxiety influences change in attentional bias to negative information. Neurostimulation methods have been shown to be effective in illuminating the causal influence of attentional control upon attentional bias to negative information (Chen et al., 2017) and could be employed to examine the impact of increasing, or decreasing, variation in state anxiety-linked inhibitory control of attention change upon attentional bias change.

Future research could also usefully investigate additional functions relationships between the variables of state anxiety, attentional control, and attentional bias to negative information. For example, given some previous theoretical frameworks proposed (Bishop \& Forster, 2013) it is plausible that state anxiety may functionally moderate the presence or strength of an association between attentional control and attentional bias to negative information. Researchers could investigate this functional relationship by adoption a methodological design that echoes the present study, and examine change in the strength of the association between these attentional processes under each state anxiety condition.

In summary, the present study demonstrated that variation in the degree to which elevated state anxiety resulted in elevation in attentional bias to negative information was predicted by variation in the degree to which elevated state anxiety served to impair inhibitory control of attention. However, contrary to expectation greater levels of state 
anxiety elicited elevation in attentional bias to negative information was predicted by lesser levels of state anxiety elicited decrement in inhibitory control of attention. These findings invite interesting speculation as to the relationship between attentional bias to negative information, attentional control, and state anxiety, and we encourage researchers to work to further illuminate this relationship. 


\section{References}

Ansari, T. L., \& Derakshan, N. (2010). Anxiety impairs inhibitory control but not volitional action control. Cognition \& Emotion, 24(2), 241-254. https://doi.org/10.1080/02699930903381531

Ansari, T. L., \& Derakshan, N. (2011). The neural correlates of impaired inhibitory control in anxiety. Neuropsychologia, 49(5), 1146-1153. https://doi.org/10.1016/j.neuropsychologia.2011.01.019

Bar-Haim, Y., Lamy, D., Pergamin, L., Bakermans-Kranenburg, M. J., \& van IJzendoorn, M. H. (2007). Threat-related attentional bias in anxious and nonanxious individuals: A metaanalytic study. Psychological Bulletin, 133(1), 1-24. https://doi.org/10.1037/00332909.133.1.1

Basanovic, J., Notebaert, L., Clarke, P. J. F., MacLeod, C., Jawinski, P., \& Chen, N. T. M. (2018). Inhibitory attentional control in anxiety: Manipulating cognitive load in an antisaccade task. PLOS ONE, 13(10), e0205720. https://doi.org/10.1371/journal.pone.0205720

Basanovic, J., Notebaert, L., Grafton, B., Hirsch, C. R., \& Clarke, P. J. F. (2017). Attentional control predicts change in bias in response to attentional bias modification. Behaviour Research and Therapy, (September). https://doi.org/10.1016/j.brat.2017.09.002

Bishop, S., Duncan, J., Brett, M., \& Lawrence, A. D. (2004). Prefrontal cortical function and anxiety: Controlling attention to threat-related stimuli. Nature Neuroscience, 7(2), 184188. https://doi.org/10.1038/nn1173

Bishop, S., \& Forster, S. (2013). Trait Anxiety, Neuroticism, and the Brain Basis of Vulnerability to Affective Disorder. The Cambridge Handbook of Human Affective Neuroscience, 553-574. https://doi.org/10.1017/cbo9780511843716.031

Chen, N. T. M., Basanovic, J., Notebaert, L., MacLeod, C., \& Clarke, P. J. F. (2017). Attentional bias mediates the effect of neurostimulation on emotional vulnerability. Journal of Psychiatric Research, 93(May), 12-19. https://doi.org/10.1016/j.jpsychires.2017.05.008

Cisler, J. M., \& Koster, E. H. W. (2011). Mechanisms of attentional biases towards threat in anxiety disorder: An integrative review. Clinical Psychology Review, 30(2), 1-29. https://doi.org/10.1016/j.cpr.2009.11.003.Mechanisms

Clarke, P. J. F., Marinovic, W., Todd, J., Basanovic, J., Chen, N. T. M., \& Notebaert, L. (2020). What is attention bias variability? Examining the potential roles of attention control and response time variability in its relationship with anxiety. Behaviour Research and Therapy, 103751. https://doi.org/10.1016/j.brat.2020.103751

Cornwell, B. R., Mueller, S. C., Kaplan, R., Grillon, C., \& Ernst, M. (2012). Anxiety, a benefit and detriment to cognition: Behavioral and magnetoencephalographic evidence from a mixed-saccade task. Brain and Cognition, 78(3), 257-267. https://doi.org/10.1016/j.bandc.2012.01.002

Derakshan, N., Ansari, T. L., Hansard, M., Shoker, L., \& Eysenck, M. W. (2009). Anxiety, inhibition, efficiency, and effectiveness: An investigation using the Antisaccade task. Experimental Psychology, 56(1), 48-55. https://doi.org/10.1027/1618-3169.56.1.48

Eysenck, M. W., Derakshan, N., Santos, R., \& Calvo, M. G. (2007). Anxiety and cognitive performance: Attentional control theory. Emotion, 7(2), 336-353. https://doi.org/10.1037/1528-3542.7.2.336

Friedman, N. P., \& Miyake, A. (2004). The Relations Among Inhibition and Interference Control Functions: A Latent-Variable Analysis. Journal of Experimental Psychology: General, 133(1), 101-135. https://doi.org/10.1037/0096-3445.133.1.101 
Godara, M., Sanchez-Lopez, A., Liefooghe, B., \& De Raedt, R. (2020). Contextual Changes Influence Attention Flexibility Towards New Goals. Cognitive Therapy and Research, 44(2), 327-344. https://doi.org/10.1007/s10608-019-10072-5

Joseph, D. L., Chan, M. Y., Heintzelman, S. J., Tay, L., Diener, E., \& Scotney, V. S. (2020). The Manipulation of Affect: A Meta-Analysis of Affect Induction Procedures. Psychological Bulletin, 146(4), 355-375. https://doi.org/10.1037/bul0000224

Kruijt, A.-W., Parsons, S., \& Fox, E. (2019). A meta-analysis of bias at baseline in RCTs of attention bias modification: No evidence for dot-probe bias towards threat in clinical anxiety and PTSD. Journal of Abnormal Psychology, 128(6), 563-573. https://doi.org/10.1037/abn0000406

Lavie, N., Hirst, A., de Fockert, J. W., \& Viding, E. (2004). Load Theory of Selective Attention and Cognitive Control. Journal of Experimental Psychology: General, 133(3), 339-354. https://doi.org/10.1037/0096-3445.133.3.339

Liu, J., Shen, K., \& Li, H. (2019). How state anxiety and attentional bias interact with each other: The moderating effect of cognitive appraisal. Attention, Perception, and Psychophysics, 81(3), 694-706. https://doi.org/10.3758/s13414-018-01650-y

MacLeod, C., Grafton, B., \& Notebaert, L. (2019). Anxiety-Linked Attentional Bias: Is It Reliable? Annual Review of Clinical Psychology, 15(1), 529-554. https://doi.org/10.1146/annurev-clinpsy-050718-095505

MacLeod, C., \& Mathews, A. (1988). Anxiety and the Allocation of Attention to Threat. The Quarterly Journal of Experimental Psychology Section A, 40(4), 653-670. https://doi.org/10.1080/14640748808402292

MacLeod, C., \& Rutherford, E. M. (1992). Anxiety and the selective processing of emotional information: Mediating roles of awareness, trait and state variables, and personal relevance of stimu. Behaviour Research and Therapy, 30(5), 479-491. https://doi.org/10.1016/0005-7967(92)90032-C

Marteau, T. M., \& Bekker, H. (1992). The development of a six-item short-form of the state scale of the Spielberger State-Trait Anxiety Inventory (STAI). British Journal of Clinical Psychology, 31(3), 301-306. https://doi.org/10.1111/j.2044-8260.1992.tb00997.x

Miyake, A., Friedman, N. P., Emerson, M. J., Witzki, A. H., Howerter, A., \& Wager, T. D. (2000). The Unity and Diversity of Executive Functions and Their Contributions to Complex "Frontal Lobe" Tasks: A Latent Variable Analysis. Cognitive Psychology, 41(1), 49-100. https://doi.org/10.1006/cogp.1999.0734

Mogg, K., \& Bradley, B. P. (1998). A cognitive-motivational analysis of anxiety. Behaviour Research and Therapy, 36(9), 809-848. https://doi.org/10.1016/S0005-7967(98)000631

Mogg, K., Bradley, B. P., De Bono, J., \& Painter, M. (1997). Time course of attentional bias for threat information in non-clinical anxiety. Behaviour Research and Therapy, 35(4), 297303. https://doi.org/10.1016/S0005-7967(96)00109-X

Mogg, K., Mathews, A., Bird, C., \& Macgregor-Morris, R. (1990). Effects of stress and anxiety on the processing of threat stimuli. Journal of Personality and Social Psychology, 59(6), 1230-1237. https://doi.org/10.1037/0022-3514.59.6.1230

Murphy, G., Groeger, J. A., \& Greene, C. M. (2016). Twenty years of load theory-Where are we now, and where should we go next? Psychonomic Bulletin and Review, 23(5), 13161340. https://doi.org/10.3758/s13423-015-0982-5

Pacheco-Unguetti, A. P., Acosta, A., Callejas, A., \& Lupiáñez, J. (2010). Attention and Anxiety: Different Attentional Functioning Under State and Trait Anxiety. Psychological Science, 
21(2), 298-304. https://doi.org/10.1177/0956797609359624

Parsons, S. (2020). Splithalf: Robust Estimates of Split Half Reliability. https://doi.org/10.6084/m9.figshare.11956746.v4

Parsons, S., Kruijt, A.-W., \& Fox, E. (2019). Psychological Science Needs a Standard Practice of Reporting the Reliability of Cognitive-Behavioral Measurements. Advances in Methods and Practices in Psychological Science, 2(4), 378-395. https://doi.org/10.1177/2515245919879695

Rudaizky, D., Basanovic, J., \& MacLeod, C. (2014). Biased attentional engagement with, and disengagement from, negative information: Independent cognitive pathways to anxiety vulnerability? Cognition and Emotion, 28(2), 245-259. https://doi.org/10.1080/02699931.2013.815154

Spielberger, C. D., Gorsuch, R. L., Lushene, R., Vagg, P. R., \& Jacobs, G. A. (1983). Manual for the State-Trait Anxiety Inventory (Form Y). Palo Alto, CA: Consulting Psychologists Press.

Van Bockstaele, B., Lamens, L., Salemink, E., Wiers, R. W., Bögels, S. M., \& Nikolaou, K. (2019). Reliability and validity of measures of attentional bias towards threat in unselected student samples: seek, but will you find? Cognition and Emotion, 0(0), 1-12. https://doi.org/10.1080/02699931.2019.1609423

Vogt, J., De Houwer, J., \& Moors, A. (2011). Unintended allocation of spatial attention to goal-relevant but not to goal-related events. Social Psychology, 42(1), 48-55. https://doi.org/10.1027/1864-9335/a000042

Vogt, J., De Houwer, J., Moors, A., Van Damme, S., \& Crombez, G. (2010). The automatic orienting of attention to goal-relevant stimuli. Acta Psychologica, 134(1), 61-69. https://doi.org/10.1016/j.actpsy.2009.12.006

Zvielli, A., Bernstein, A., \& Koster, E. H. W. (2015). Temporal Dynamics of Attentional Bias. Clinical Psychological Science, 3(5), 772-788. https://doi.org/10.1177/2167702614551572 
Table 1

Descriptive statistics of measures of state anxiety, attentional bias, and attentional control, under each state anxiety manipulation condition for each trait anxiety group. M (SD).

Measure

Pre State Anxiety Induction

Post State Anxiety Induction

\begin{tabular}{cccc}
\hline Low Trait Anxiety & High Trait Anxiety & Low Trait Anxiety & High Trait Anxiety \\
\hline 51.25 & 92.58 & 72.46 & 108.28 \\
$(28.34)$ & $(36.39)$ & $(41.40)$ & $(37.03)$ \\
1.26 & 6.09 & -4.46 & 3.97 \\
$(12.97)$ & $(20.06)$ & $(21.94)$ & $(20.48)$ \\
101.57 & 81.15 & 115.78 & 104.84 \\
$(82.15)$ & $(74.83)$ & $(94.36)$ & $(75.03)$
\end{tabular}

State Anxiety Index

Attentional Bias Index

Inhibitory Attentional

Control Cost Index
101.57

$(74.83)$

(94.36) 


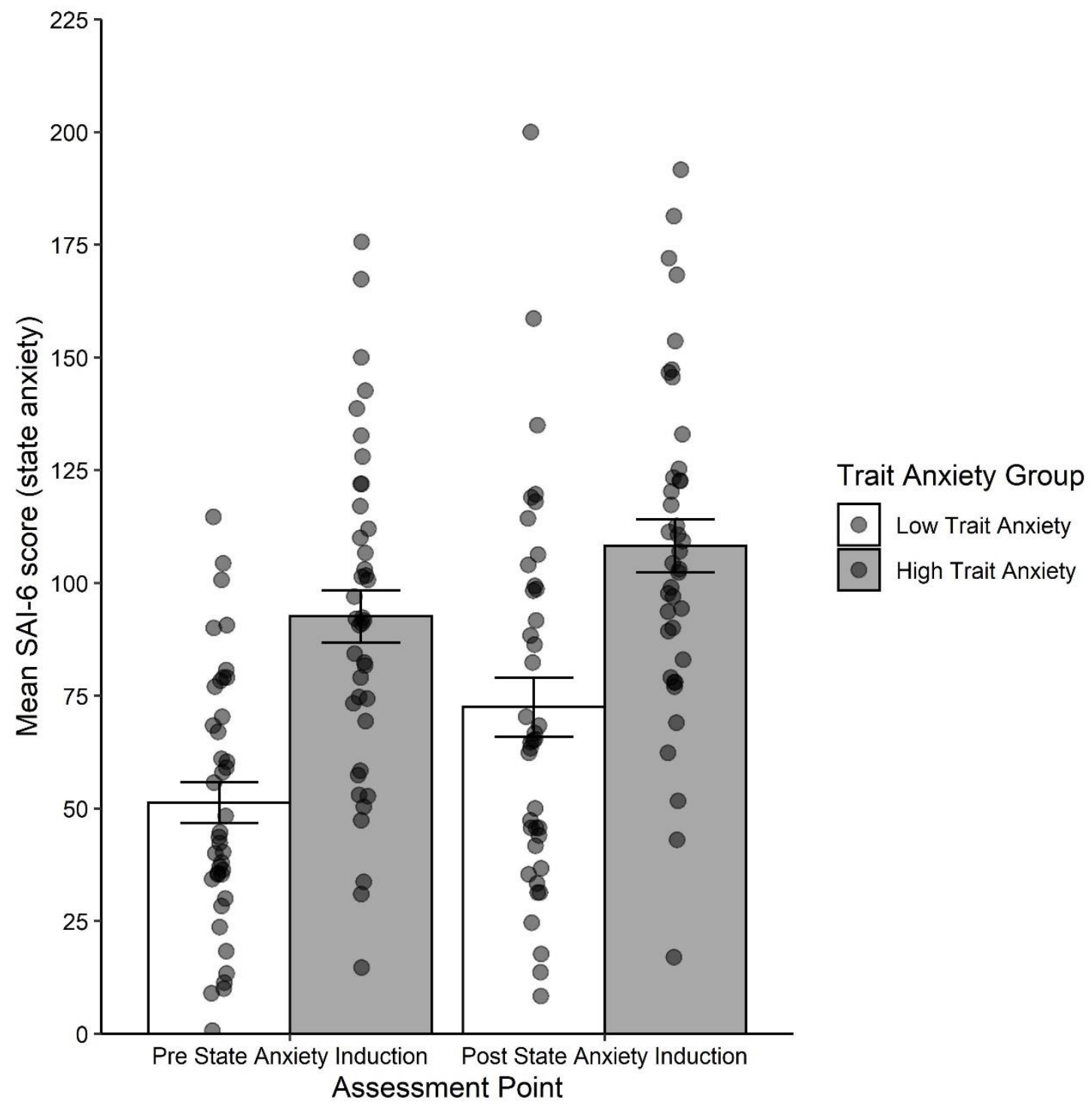

Figure 1. Mean SAI-6 scores, reflecting level of state anxiety, for each assessment point and trait anxiety group. Height of bars represent mean latencies, error bars represent standard error, points represent mean scores of individual participants. 


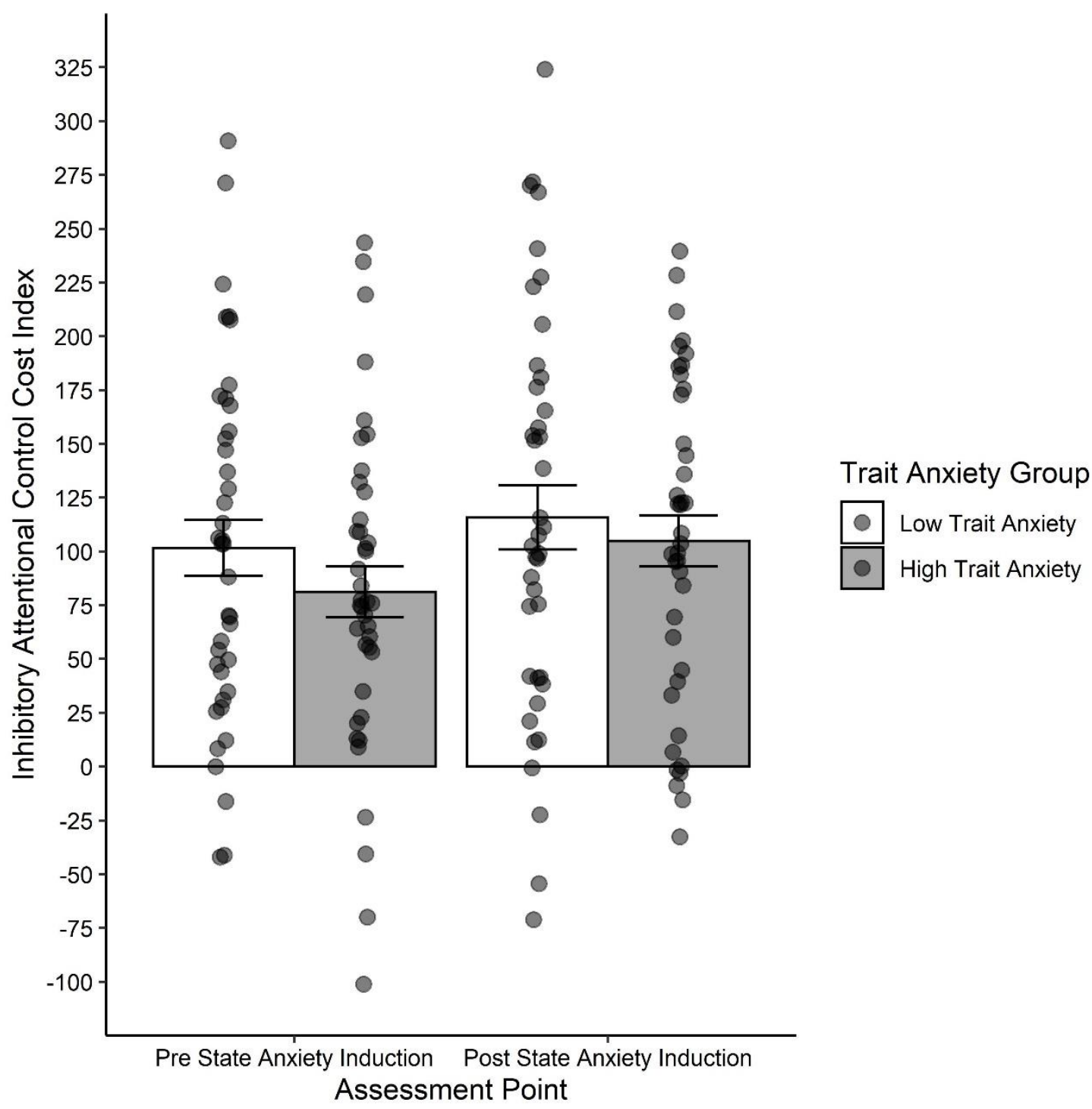

Figure 2. Attentional Control Index scores for each assessment point and trait anxiety group. Height of bars represent mean index score, error bars represent standard error, points represent index of individual participants. 


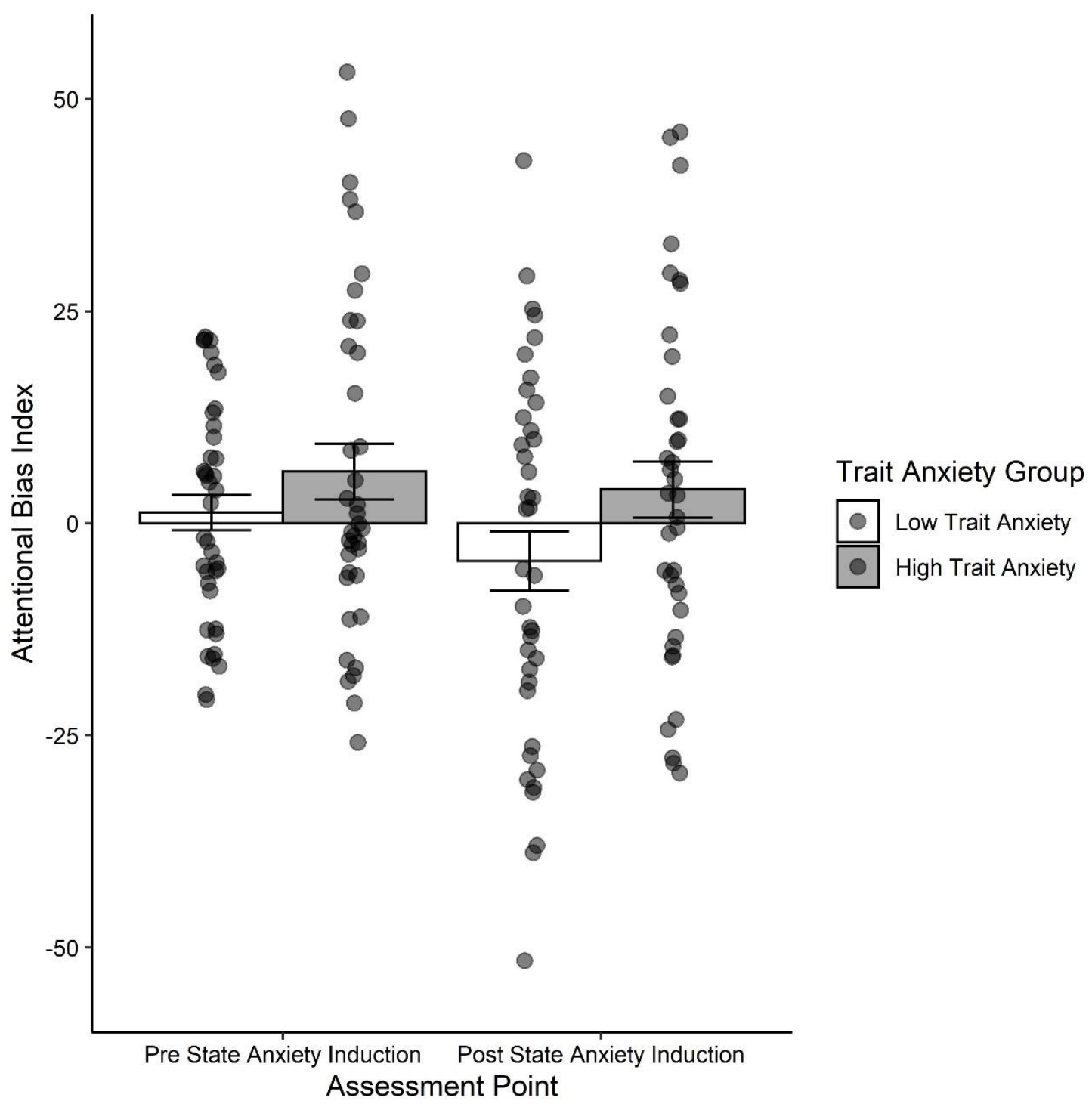

Figure 3. Attentional Bias Index scores for each assessment point and trait anxiety group.

Height of bars represent mean index score, error bars represent standard error, points represent index of individual participants. 


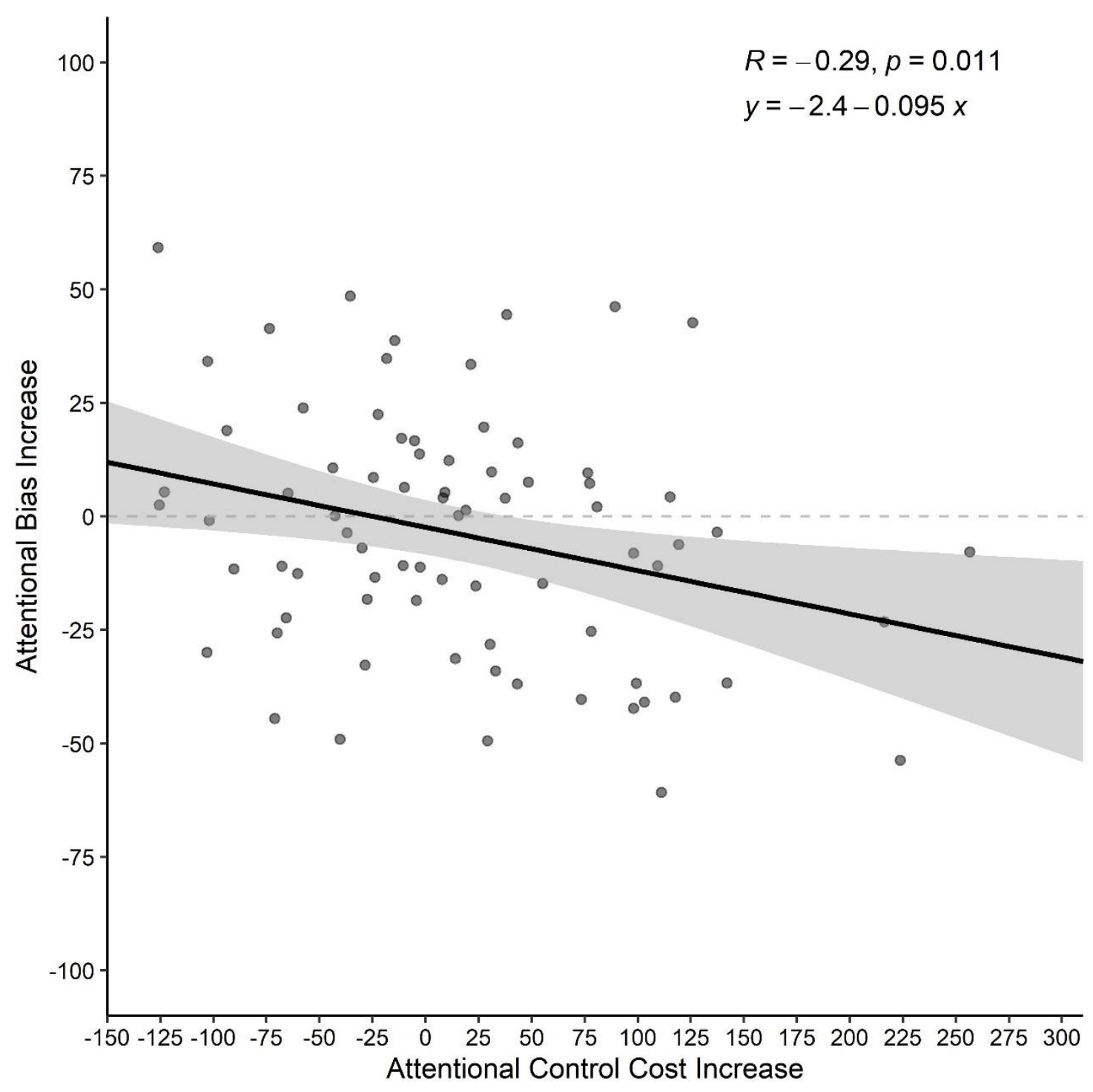

Figure 4. Association between increase in attentional bias index scores and increase in

attentional control index scores across participants. Line represents line of best fit, shaded area represents standard error, points represent individual participants. 